\title{
THE TREATMENT OF DECAYED WOOD FROM DEAD TREMBLING ASPEN TREES FOR GROWTH-RING ANALYSIS ${ }^{1}$ \\ BY ARTHUR W. GHENT ${ }^{2}$
}

Upon graduation from the University of Toronto in 1950 with the degree of B.Sc.F., Artbur W. Ghent joined the Forest Insect Laboratory in Sault Ste. Marie, undertaking research projects on stand decadence and regeneration in relation to spruce budworm outbreaks. He was awarded the degree of Master of Arts in the spring of 1954 for graduate studies in the Zoology Department of the University of Toronto on the gregarious feeding behavior of the jack pine sawfly. During graduate training, he has been awarded the Robert W. Lyons Fellowship in Forestry and an Ontario Research Council Scholarship.

\section{ABSTRACT}

This paper elaborates certain procedures briefly outlined in an earlier article. Although dry wood from dead trembling aspen has been found best suited to growtb-ring analysis, in its absence use bas been made of watersaturated wood from the underside of fallen trunks. A technique of wax impregnation, adaptable to wood in botb early and advanced stages of decay, is described. Rapid impregnation with near-boiling wax is sufficient to facilitate transverse sectioning of relatively sound wood; advanced decays require a slower impregnation with cooler paraffin. Hot xylol is used to remove the wax and flatten the section. Successive, overlapped sections provide an extended series of measurements to establish the orientation in time of mortality. Incomplete rings for the years just prior to death are checked by measurement of the outer growth at additional points.

\section{INTRODUCTION}

In an earlier paper (1), devoted largely to the statistical aspects of cross-dating ring patterns between living and dead trees, brief reference was made to a wax impregnation procedure for the preparation of study material from bady deteriorated trembling aspen, Populus tremuloides Michx. Through the extended use of such decayed material, certain refinements have been effected in this and related procedures, and these are now presented.

\section{Collection}

\section{METHODS}

In practice, complete discs from dead trees have been found most desirable for study, but partial discs or even small fragments of peripheral wood must frequently be accepted. Dead trembling aspen broken in mid-trunk may remain standing as dry stubs for 15 or more years with only minor deterioration, and from these, complete discs can usually be obtained. More difficult to study are those which have been uprooted, or wind-broken close to the ground. In such instances, advantage has been taken of one or other of two extremes in moisture conditions. Parts of the trunk supported clear

1 Contribution No. 125 Forest Biology Division, Science Service, Department of Agricaltare, 2 Forest Insect Laboratory, Sault Ste. Marie, Ontario. 
of the ground by other debris remain dry and relatively sound for many years. Similarly, any point on the trunk where the bark has sloughed away may provide a partial disc of sound wood, particularly if that point is exposed to the sun. At the other extreme, wood on the under side of fallen trunks may be in such close contact with the moist humus that it remains water saturated. When no other material is available, fragments of such wood may provide several inches of measurable rings for analysis. Within the intermediate moisture range, poplar wood deteriorates rapidly.

\section{Preliminary preparation}

The system of checks on the accuracy of ring dating previously described (1), based on prominent rings traced on the study disc from one radius to the next, has been adopted as standard practice during the preliminary preparation of discs cut from living trees. All subsequent analysis of tree mortality depends on the absolute accuracy of this dating. However, it is impossible to apply such checks to badly decayed partial discs or peripheral fragments, since these afford only isolated islands of wood in which growth rings can be measured. This limitation has necessitated the development of a technique of preparation and later analysis better suited to such material.

When available, as many as five or six of these perpheral islands of measurable wood are cut as small wedges from the study material, one or two being cut sufficiently deeply to include the 55 to 60 rings desirable for statistically reliable cross-dating. Later sectioning of these wedges can be performed most readily if they are of a uniform degree of deterioration throughout. Growth rings can usually be distinguished even at an advanced stage of the common brown cubical rots of poplar since the gross cellular structure remains virtually unmodified in appearance.

\section{Impregnation with paraffin}

It has been found necessary to impregnate these wedges with paraffin wax to prevent even slightly decayed wood from crumbling during transverse sectioning. Commercial "Parowax" prepared for home preserving is suitable and inexpensive. A bath of molten paraffin, roughly an inch deep, is heated until the first indications of fuming are observed. At this point, the wedges are stood on transverse end in the bath, with the end more favorable for examination downwards. The time required for adequate penetration was originally given (1) as a "half hour", but this has been found to be in excess of what is generally required. With near-boiling wax, there is an immediate and vigorous release of moisture vapor from the wood, which for this reason should be air-dried for several days prior to treatment. After four or five minutes, penetration of wax to a depth of several inches is obtained.

Should the study material be only slightly decayed, this rapid, hightemperature impregnation is sufficient, and the wedges may be removed from the bath and allowed to cool. Should the decay have reached a more advanced stage in which the wood crumbles if cut or sawn, further impregnation is required for satisfactory results. Leaving the wedges in the bath but removing the bath from the source of heat, the wax is allowed to cool until just abova the melting point. Wedges so treated carry a noticeably heavier load of wax 
than those given only high-temperature impregnation, and are virtually without air spaces. By this simple expedient, the one impregnation procedure can be adapted to wood in either early or advanced stages of decay.

Fragments of wood taken from the water-saturated underside of fallen trunks may require special treatment. These fragments are generally so fragile that they are best left intact as collected until paraffin impregnation is complete. Prior to collection, the convex outer surface of such fragments presses directly against the moist humus. So positioned, the peripheral surface remains smooth and retains the complete ring record of the tree's final years of growth, until the process of decay and disintegration is complete. Gradually refined technique applied to dead trembling aspen in the Lake Nipigon region of Ontario, indicates that this process requires a period slightly in excess of 30 years from the time of death of the tree.

The opposite surface of these fragments, facing towards the now disintegrated core of the tree, is often irregular, the high points tending to break off despite careful handling. Since such material usually provides relatively few rings, it is necessary to protect these high points to retain sufficient rings for statistically reliable cross-dating. Following the high- and low-temperature impregnation already described, the almost solidified wax is ladled on to this irregular surface until a protecting shell is formed. After the wax has solidified, the fragment is sawn transversely through the most prominent of the high points, thereby exposing two similar surfaces from which slides may be prepared.

\section{Preparation of Slides}

To prepare slides for growth-ring measurement, the study wedges can be sectioned most satisfactorily with a hand knife. The size of the wedge needed to include an adequate series of rings precludes the use of microtome sectioning. A good quality knife sharpened to a razor edge is necessary for best results. Badly decayed aspen wood, even following wax impregnation, tends to shatter when cut. This tendency is greatly reduced if wedge and knife are so oriented that the cutting edge describes a radial sweep as if pivoted at the core of the tree; in the same manner as the hand of a clock. The relatively thick sections obtained with a hand knife are often curled, and must be cleared of wax and flattened for microscopic examination. Hot xylol, heated in a near-boiling water bath, has been used to remove the wax and flatten the section. Using a small pipette, the solvent should be allowed to run slowly on to the slide to one side of the study section, since a sudden gush of hot xylol will shatter sections from badly decayed wood.

\section{Ring measurement and analysis}

Starting from the outside of the wedge, successive overlapping sections are taken working towards the core. The overlap must be sufficient to ensure unquestionable relocation of ring numbers by inspection and re-measurement. Frequently one must be satisfied with ten or fewer new rings per section. Each section is most efficiently measured as it is taken. Temporary slides of 
this type, when viewed by transmitted light, allow precise micrometer settings to be made on the thin line of terminal parenchymatous tissue laid down in trembling aspen.

Measurements on at least one wedge are carried back 55 to 60 years, or to the earliest measurable ring if fewer are available. The outer 15 to 20 years' growth on the additional wedges is then measured as a check against missing rings in the years just prior to death. Measurement of 15 to 20 rings is usually adequate to ensure the correct alignment of the short with the extended series, since agreement within one or two years is to be expected. In practice this alignment can be effected either by direct examination and comparison of the measurements for the different wedges, or should inspection leave room for doubt, by rough plotting of the measurements.

Growth curves for trees analysed in this manner are, therefore, composed of two parts. The curve for the outer 15 to 20 rings represents the average of four or five radii thus supplying the critical accuracy of ten required to define the last year of growth. The remaining 40 to 45 years of the curve represent one series of measurements from a more vigorous period in the growth of the tree, for which this small sample suffices. This series, by supplying the length to the growth curve required for statistically reliable cross-dating, serves to establish the orientation in time of mortality.

\section{REFERENCE}

(1) GHENT, A. W., 1952; "A technique for determining the year of the outside ring of dead trees". For. Chron. 28, (4): 85-93. 